\title{
Inclusive Playgrounds: A Reality or a Utopia in Our
}

\section{Cities?}

\author{
Michela Dalprà \\ Department of Civil, Environmental and Mechanical Engineering (DICAM), University of Trento, Trento 38123, Italy
}

\begin{abstract}
The culture of inclusion needs to be continuously promoted, supported, strengthened and tested to remove the barriers and deficiencies still present in the built environment around us. Do the play areas in parks, gardens and squares offer real opportunities for persons with different abilities to play and have fun? Do they promote the principle of integration and create opportunities for inclusion? To answer these questions, an audit tool has been developed to assess the inclusivity of outdoor play areas in the central and suburban areas of our cities. The key elements of the evaluation focus on the physical environment of the playground and are grouped in three thematic areas: physical and sense-perception accessibility; accessibility to the experience and participation and socialisation. Although children's right to play is now recognised and there is much talk of inclusion, application of the tool to an Italian city has shown that the right to play is not fully and satisfactorily applied. Inclusive playgrounds are still not frequent enough and a new approach to their design is needed.
\end{abstract}

Key words: Playground, inclusion, accessibility, children with disabilities.

\section{Introduction}

In both the central and most peripheral areas, our cities need welcoming and better quality meeting places where all children and their families can play, have fun and participate in cultural and recreational life. The exercise of play is an irreplaceable and inalienable human activity [1]. The right to play and recreational activities is a fundamental right for children enshrined in Article 31 of the United Nations Convention on the Rights of the Child (1989). Play and sport are, in fact, recognized as activities that advance and develop the sociality and well-being of all children. According to the United Nations Convention on the Rights of Persons with Disabilities (2006), play must also be guaranteed to children with different types of abilities as expressed in Art. 30, in a logic of inclusion, equality and equal opportunities.

In order to develop their potential and lay the foundations for healthy and happy growth, every child

Corresponding author: Michela Dalprà, professor, research fields: architectural design, built environment accessibility, and sustainable building assessment. has a need and right to prove themselves through play, in all its aspects and declinations [2]. Numerous studies have shown that "children with disabilities wish to become part of the world of children and playing with other children is the way to enter that world" [3]. All children with disabilities "want to play in one way or another" [4].

The right of children with disabilities to play and sport is not fully respected today. The barriers that hinder and/or limit children in play may be institutional, socio-cultural or physical.

Are our way of life and our cities today able to guarantee Children of Different Abilities (CoDA) the right to play? Do children have sufficient time and space to play outside the home? Do inclusive playgrounds exist where they can meet friends or peers and play together without barriers or discrimination?

A playground is a space equipped to play, exercise and be together. It is a generally outdoor area with free access designed for recreational activities for children of different age groups. It can be created inside public gardens, squares, schools, beaches, 
shopping centres or holiday accommodation facilities. If the play area is "well designed it can become a valuable vehicle for integration, allowing children to play outdoors, experience their own autonomy in a protected environment, socialise, get to know other children and other families and gain first-hand experience of environments other than that of the family" [5].

Playgrounds can create an environment of inclusion or exclusion [6]. The inclusive playground is not a "special" playground for children with disabilities [7] or a space where there are one or more games for the exclusive use of children with disabilities, just as the disabled toilets in buildings are not inclusive. An inclusive playground is something different. An inclusive playground is "purpose-designed to include all members of the community (irrespective of age, ability or any other perceivable difference) in the experience of play in a playground" [8]. It is a barrier-free space where persons with different physical, sensory and intellectual abilities can play, experiment, move about and enjoy themselves safely, interacting with each other. Children have different social, play, communication and movement needs according to their age and state of health. Equipment for an inclusive playground should be designed, built and installed to provide opportunities for independent or assisted play for the majority of children with motor, sensory (sight-hearing) and cognitive disabilities. The overall goal of the inclusive playground is "to provide all children a rich playground environment for play and quality social participation" [9]. The inclusive playground is an area that welcomes not only children, but also parents and family members with or without disabilities. Both must have access to the play area and adults must have the pleasure of playing with their children, grandchildren or younger siblings [10].

Inclusive playgrounds are also starting to be a reality in Italy. More or less virtuous examples created in recent years in some Italian cities (e.g. Milan, Rimini, etc.) can be found alongside many playgrounds that need to be improved and integrated to make them truly accessible and usable for all children, including those with special needs [11].

This article gives a summary of a study mapping the inclusivity of play areas in the city of Trento (TN), capital of the Autonomous Province of Trento (Italy).

\section{Methodology}

An audit tool has been developed to evaluate and construct a map of inclusion in play areas. This tool makes it possible to highlight the strengths and/or major critical points that limit the experience of playing and relaxing for persons of different abilities in outdoor playgrounds in the urban and peripheral contexts of our cities. The study was divided into two distinct phases: analysis of inclusivity in play areas and its actual presence in the field.

In the first phase, after exploring the meaning of an inclusive playground through a review of literature, the key elements of the evaluation were identified and grouped in three thematic areas:

- physical and sense-perception accessibility: the possibility for everyone to have access to the spaces, move about independently in them and reach the play and rest areas in an equal, inclusive, independent and safe way;

- access to the experience: an opportunity for everyone to understand and live the experience of the game in all its variety, in other words, the possibility for everyone to have experiences, try and experiment;

- participation and socialisation: the possibility for children and adults to socialise, participate and share during the play activities and also in the rest/relax areas.

An analysis sheet was subsequently developed to explore the existing outdoor play areas with the aim of verifying the actual presence of abundant play equipment offering opportunities for $\mathrm{CoDA}$ for reasons of age, disability or health conditions.

The analysis sheet takes its cue from the OPEN protocol survey sheet for mapping barriers limiting the 
accessibility of nature trails and parks and includes new items.

The sheet is divided into five sections and examines the main environmental factors able to promote inclusion, grouped into the thematic areas mentioned above.

The first section (Identification Elements) collects general information about the play area, such as name, location, type, ownership.

The second section (Accessibility) analyses accessibility and access to the playground, internal mobility, the surface of the paths and the surface around and under the play and rest facilities. The objective is to verify that children and adults can arrive, enter, orient themselves, move about and approach the equipment.

The third section (Play Experience) examines the play dimension by identifying the different types of play experience available to CoDA. The aim is to verify the quality of the recreational experience by proposing elements of play on the ground and composite equipment capable of stimulating activities for physical, dramatic, sensory, cognitive and creative play.

The fourth section (Participation and Socialisation) verifies whether the playground includes equipment that can create opportunities for shared play, in other words, equipment that promotes parallel and cooperative play and can be used by CoDA. The aim is to verify whether the park limits or prevents participation and socialisation in relation to disabilities.

In the fifth section (Rest and Relaxation Experience) we analyse the elements of furniture (e.g. benches, tables, etc.) and service facilities (e.g. fountains, waste bins, toilets, etc.) typically present in the rest areas to verify their use by persons with disabilities.

In the second phase the analysis sheet was applied in the field to ten playgrounds in and around Trento. The case studies were selected from the Parks and Gardens database of the Municipality of Trento on the basis of the following criteria: (i) play areas created or upgraded in the last decade (2010-2020) and (ii) play areas advertised as "accessible", as containing "accessible games" or as "playgrounds for all" on the playground's website, in articles in the local press, etc.

\section{Results}

The analysis conducted provides an albeit partial and not exhaustive picture of inclusiveness in play areas in and around Trento. In particular, the paper provides a summary divided according to the previously mentioned thematic areas of the evaluation, highlighting strengths and weaknesses mainly attributable to incorrect choices of materials and equipment during design and construction, as well as in some cases a lack of effective maintenance.

\subsection{Physical and Sense-Perception Accessibility}

The inclusive playground must first and foremost be accessible to children and adults with and without disabilities.

All the playgrounds examined are easy to reach and guarantee access to persons with different abilities. Those with their own parking have at least one parking space reserved for persons with disabilities.

Although with accessible paths connecting the play areas, the games in many of the surveyed playgrounds are located on surfaces that are not accessible, in other words, they cannot be crossed with mobility aids (e.g. wheelchair, pushchair, walkers, etc.). This is the case, for example, of the play unit in Fig. 1 in a newly built playground near an expanding residential area of Trento. It is a miniature house for theatre and music designed by the producer to offer CoDA opportunities for dramatic, exploratory and collaborative play. Its location on sand, however, makes the area of use inaccessible. The sand prevents the wheelchair-bound child from reaching the play area, moving around and enjoying the experience of playing alone or together with other children.

Only $20 \%$ of the playgrounds analysed have accessible anti-trauma cast rubber flooring around and 
underneath the play equipment, rather than bark, grass, gravel or sand.

Another criticality identified regards the impossibility of easily reaching and using the rest areas. Picnic areas are often equipped with benches and tables located on unsuitable surfaces, namely surfaces that are not perfectly flat and on the same level (Fig. 2). The tables are almost always unsuitable for wheelchair users (Fig. 3); only one playground was found to have benches and a child-friendly table (Fig. 4).

Benches with a backrest are almost always installed along the paths. However, they are rarely equipped with armrests and located in areas with a perfectly level and even surface at the sides to facilitate the presence of pushchairs or wheelchairs alongside.

Almost all the playgrounds examined have drinking water fountains, not always accessible to wheelchair users. The greatest criticality regards the water collection grid, in many cases not flush with the ground. A double-height fountain has been installed in only one playground.

Waste containers are always present in different shapes and sizes, with and without a lid and correctly positioned so as not to constitute an obstacle. At most of the time they can be used by children and wheelchair users as they are of a suitable height.
For people with sensory limitations, the barriers consist in a lack of clear and correct indications (visual, tactile and acoustic), not only to signal the presence of any obstacles or sources of danger, but also to communicate the organisation of the area and distribution of play and service facilities (e.g. rest areas, waste bins, drinking water fountains, etc.).

With one exception, all the play areas analysed are without artificial aids (e.g. tactile tracks, tactile signs, voice alerts, relief maps, handrails, etc.) to help orient visually impaired or blind persons. Only the "Enzo Tortora" park includes light grey asphalt paths which contrast well with the surrounding greenery and the colours of the play areas (Fig. 5), together with tactile indications on the ground to indicate the presence of rest areas (Fig. 6).

\subsection{Play Experience}

Access to the play experience refers to the concept of the usability of play equipment which should be usable by the widest possible number of people with and without disabilities. Having access to the play experience means hardly ever being a spectator, but having the chance to have not just one, but several different play experiences. The inclusive playground must offer a full range of equipment with various play values and challenge levels. In particular, the

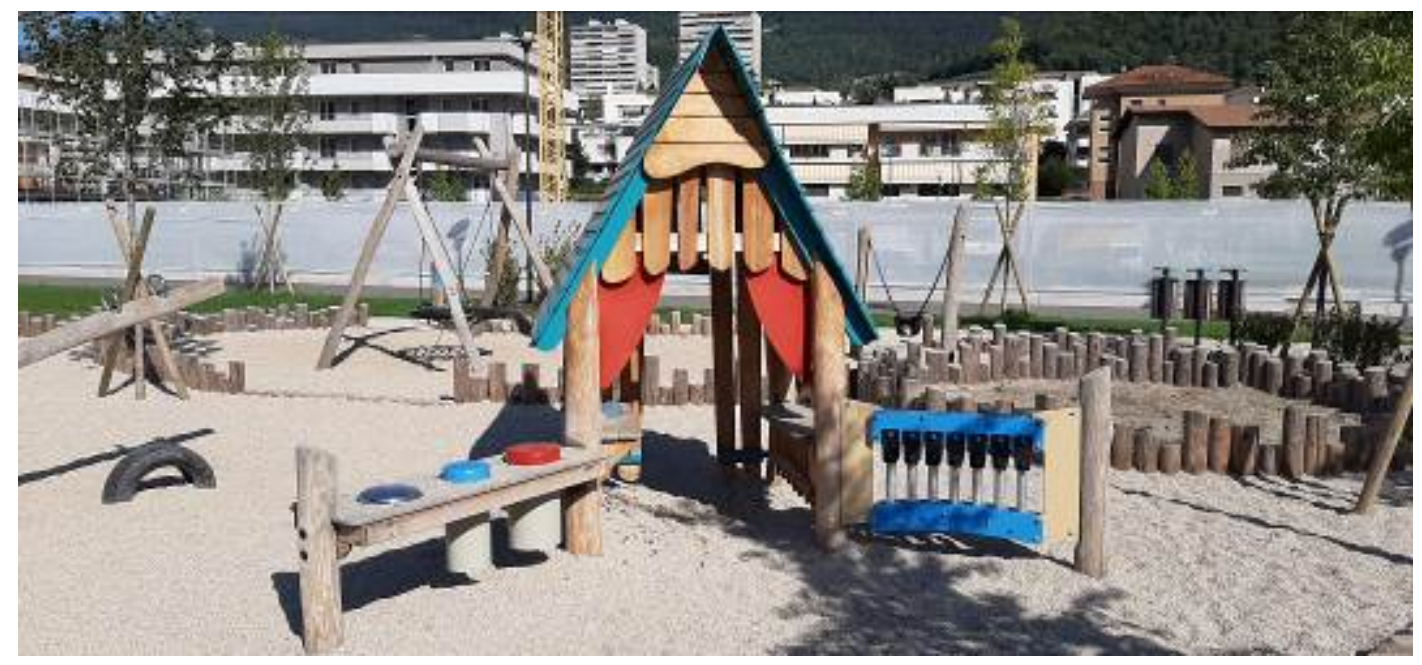

Fig. 1 Example of a thematic game on the ground with inaccessible area of use. 


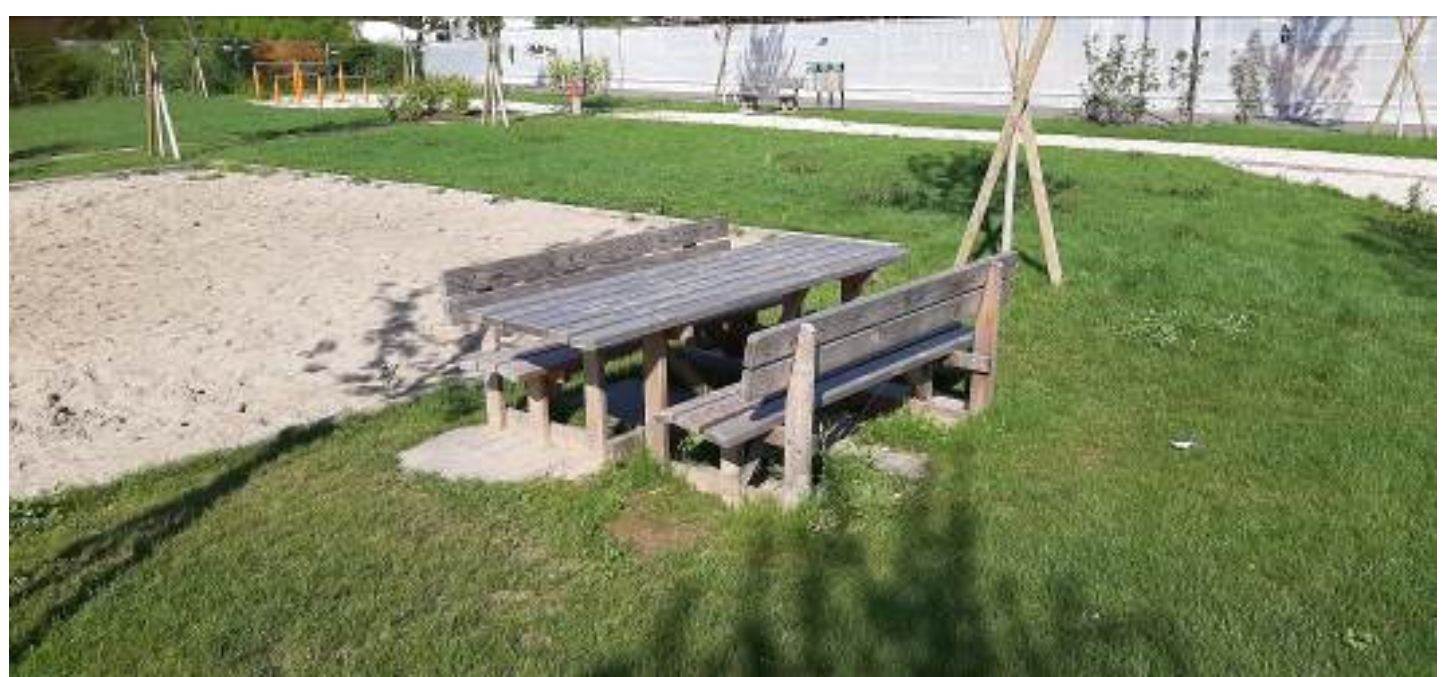

Fig. 2 Example of a picnic area on a surface which is not flat and even, equipped with furniture that can not be used by a wheelchair user.

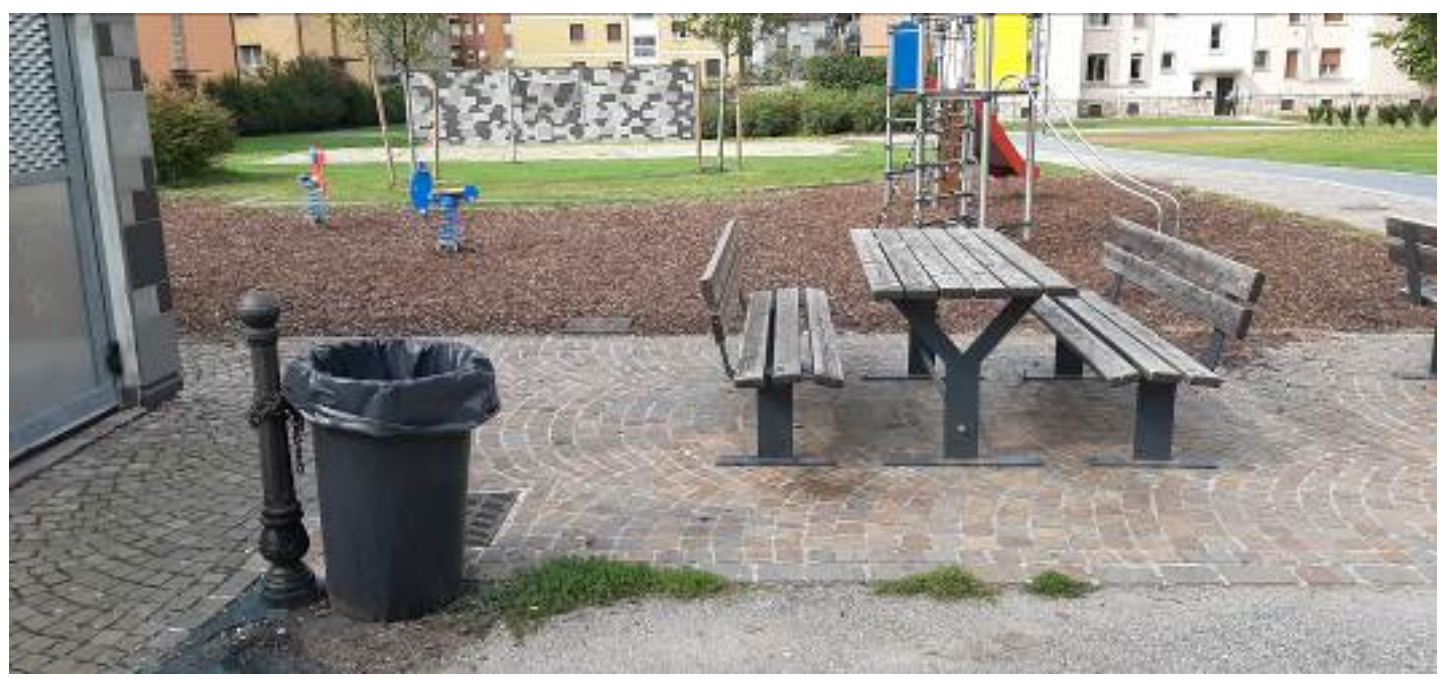

Fig. 3 Example of a table on a flat even surface, but not usable by a wheelchair user.

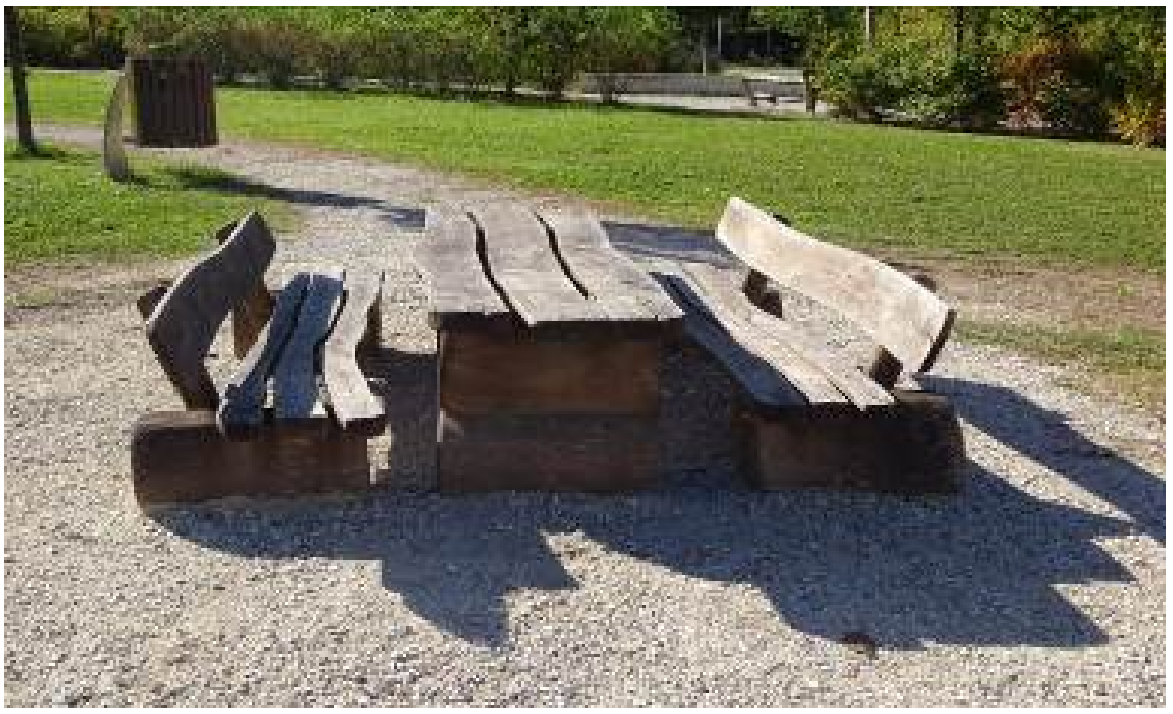

Fig. 4 Example of a table with child-friendly benches. 


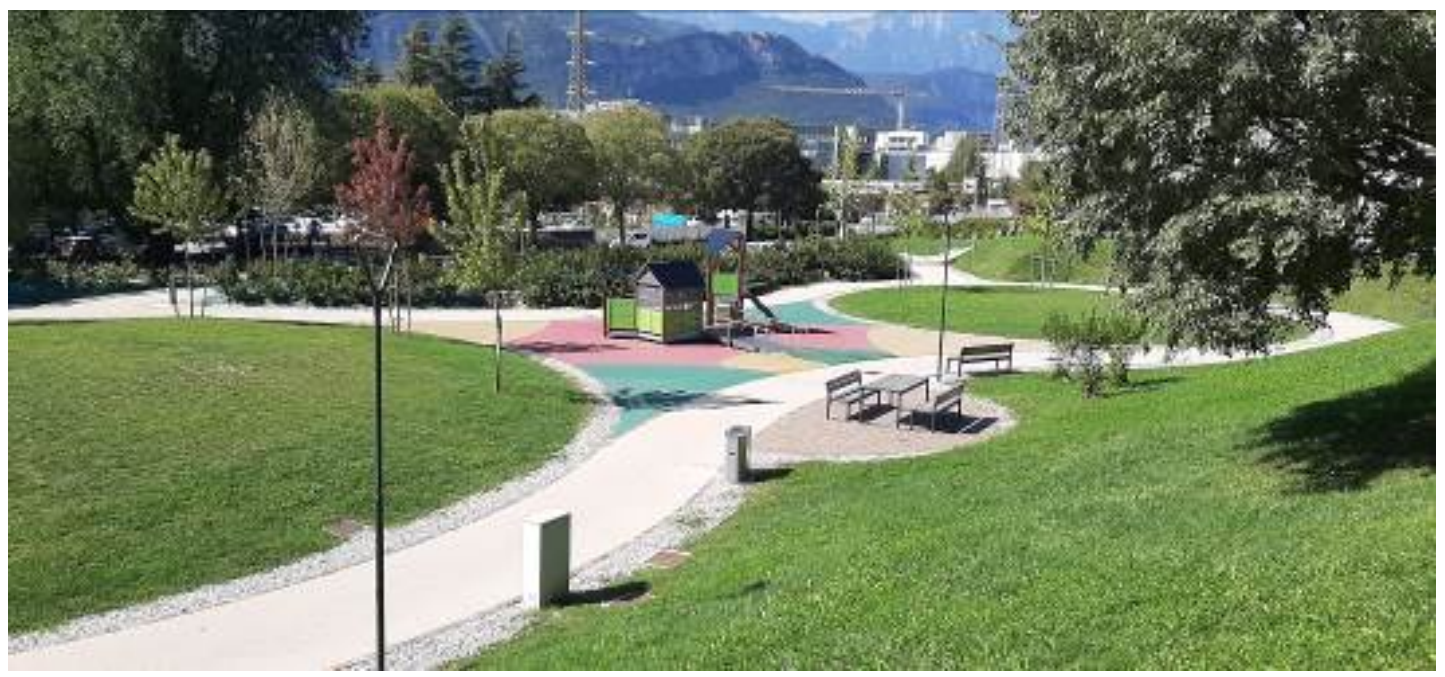

Fig. 5 Example of connecting paths and play areas paved in a contrasting colour.

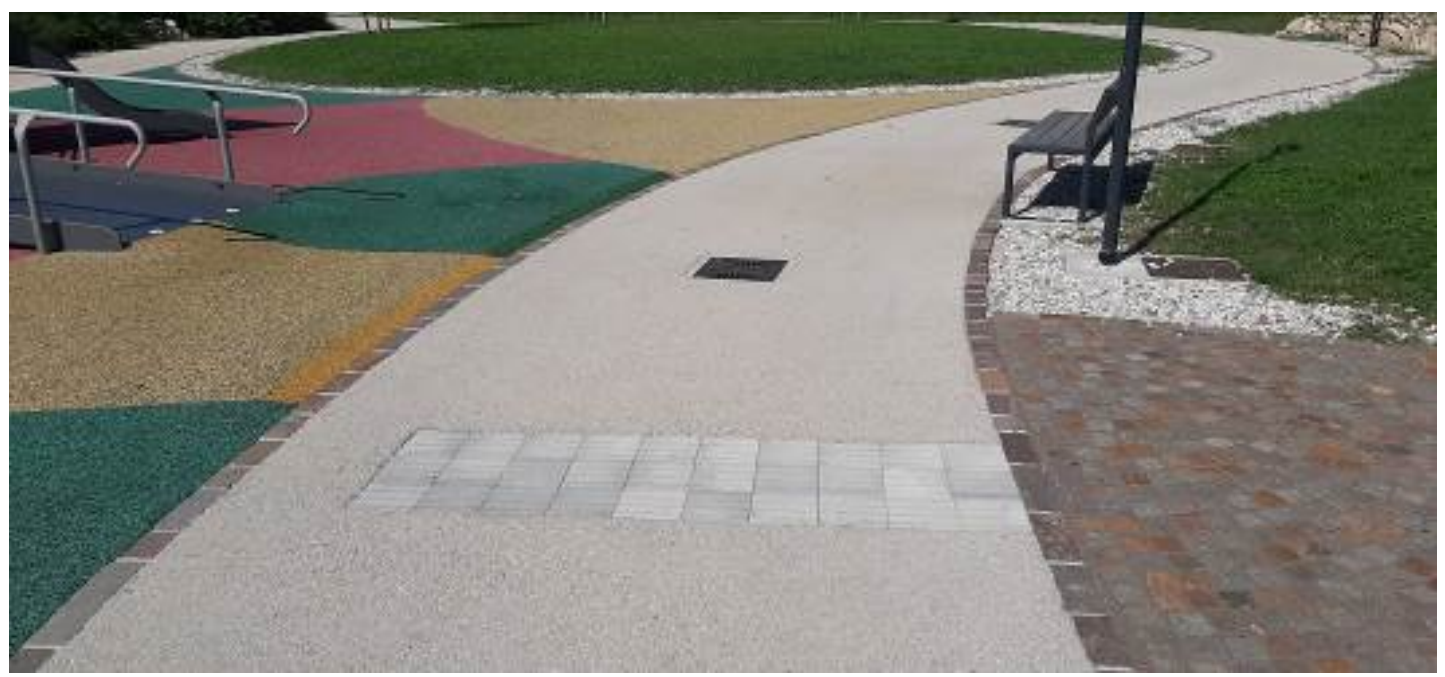

Fig. 6 Example of a tactile ground-level indication.

playground should offer CoDA physical, dramatic, cognitive/creative and sensory play experiences, mainly to be enjoyed in total autonomy or with the support of a family member or companion.

For CoDA, the offer of play experiences in playgrounds in and around Trento is quite limited and almost always associated with the physical game represented: (a) by a basket swing, or swing with seats equipped with protective elements; (b) by a carousel with base platform flush with the underlying ground; (c) by a spring game with backrest and side protections. Climbing facilities and slides cannot be used by all children. Themed ground-level games are rare. There are no composite structures with accessible routes to enable wheelchair users to reach the raised components. Games for handling and kneading with sand and water are either absent or, when present, unusable for children in wheelchairs or sitting down.

There is also a lack of games and paths with sensory stimulation (sound, optical and visual). There are no opportunities to explore tactile experiences and scent. Only one playground included sound and music games that offer important stimuli to children with visual disabilities or intellectual difficulties. The lack of sensory experiences penalises all children, not only those with sensory and cognitive disabilities.

All playgrounds include open spaces for fantasy play and those located in public gardens provide 


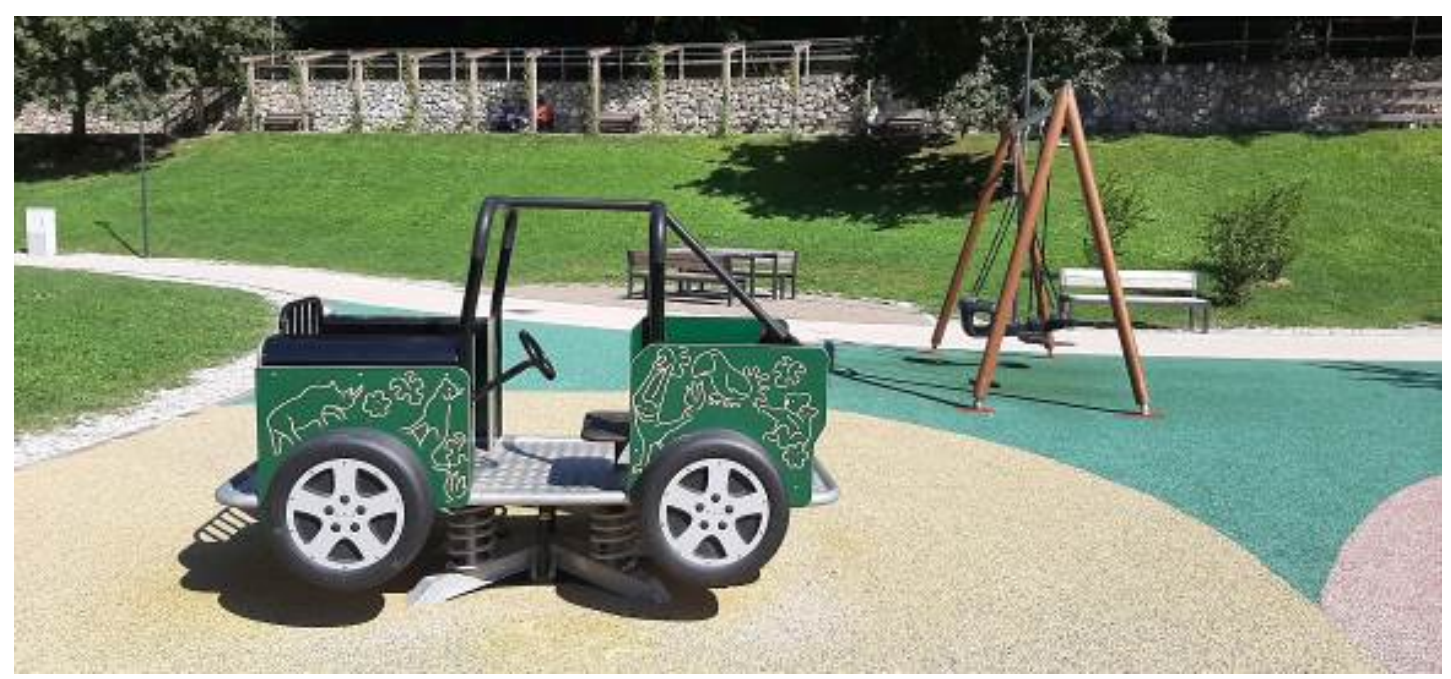

Fig. 7 Example of a multi-seat spring game.

opportunities to play with nature and natural elements (e.g. trees, grass, flowers, etc.).

\subsection{Participation and Socialisation}

Social play facilities and rest areas allow children and adults to get to know each other, share experiences and remove relational barriers. The benefits deriving from group and parallel play help the children develop communication skills and the way in which they relate to others in an environment different from that of the family and school.

In the playgrounds examined, the only equipment offering parallel play opportunities for CoDA is the double swings with different seats, giving peace of mind and making the movement safe. There were no coupled or double-track slides, no see-saws usable by CoDA and no other types of game. As far as group play equipment is concerned, a basket/nest swing was present in almost all playgrounds. This allows several CoDA to swing safely and socialise, but in many cases it was installed on an inaccessible surface. This type of game is often accompanied by small groups of single-seat spring games, some with side and back protection, but always installed in inaccessible areas of use. In just one case, the spring game was multi-seat and installed on a suitable surface (Fig. 7).

\section{Discussion}

At international, national and also local level, the progress made in designing and constructing play areas for everyone must be acknowledged. However, the right to play is not always fully and satisfactorily applied. Unfortunately, there is still little thought given to children and persons with disabilities.

The analysis shows that truly inclusive playgrounds are still quite rare. The play areas in and around Trento offer CoDA a limited play experience. No playground had on-site panels providing information about organisation. In many cases, the equipment is inaccessible due to an unsuitable surface around and underneath. An inclusive playground is not merely a collection of play equipment designed and declared to be for all children. Although the play equipment may be designed according to Universal Design principles, this does not ensure that the playground in which it is located is truly inclusive.

The survey confirmed the findings in other studies that "conventional playgrounds are unlikely to be inclusive" [8]. To improve the quality of playgrounds, we need more culture and a comprehensive view of the needs and well-being of playground users.

The inclusive playground does not result from a designer's action alone. The inclusive playground project is a demanding challenge to be tackled with an 
approach based on dialogue and interdisciplinary input. The design process must be collective, participatory and aimed at identifying new opportunities and points of view [12]. The concept of inclusion should guide designers to conceive the whole area as an integrated space to develop physical, cognitive, emotional and social skills. Designers must investigate the needs of children with disabilities and the requirements of their caregivers in order to identify facilities that effectively meet their needs [13]. Guaranteeing children with disabilities the right to play "implies a commitment that embraces a number of actors and a number of domains" [14]. The creation of an inclusive playground is the result of the collaboration of a variety of stakeholders such as city planners, designers, local governments, academic researchers, child health-care professionals, educators and parent groups. Children should also not be forgotten in order to better understand their needs and desires [15]. Important decisions are often made by people who do not use or frequent playgrounds, in the belief that they are able to interpret what attracts and pleases children [16].

Another key figure in the design of inclusive playgrounds is the occupational therapist that has the skills necessary to assess people's environment and needs and to identify barriers and factors which facilitate and meet the needs of persons with and without disabilities [17]. Occupational therapists are, in fact, ideally placed to defend children's right to play, combining knowledge of environmental barriers with an understanding of disability and a specific knowledge of occupation [18].

Although the scientific literature of the last decade highlights the importance of interdisciplinary and participatory design, our cities still offer few examples of inclusive playgrounds that show evidence of the contribution of experts. There is therefore still a great deal to be done. The culture of inclusion needs to be continuously promoted, supported, tested and strengthened in order to remove the barriers and deficiencies still present in the built environment around us.

\section{Conclusions}

The importance of inclusive play is in theory recognized by all, but in practice a new culture of play for CoDA is needed.

Action must be taken as soon as possible to provide a rapid and well-informed response to the right to play for all. While in other countries, standards and guidelines have been developed to support sector designers and operators in the creation of accessible and inclusive playgrounds, in Italy there are no technical specifications and regulations for inclusive play areas.

We currently have examples of good and bad practice in inclusive playgrounds. Although each project is unique and characterised by specificities linked to its own context, the analysis of best and worst practices is not only a starting point to work on the creation of effective project models for inclusive play areas, but also a tool to collect information that could be useful for the elaboration of plans to implement interventions to achieve the accessibility and usability of urban places (e.g. plans to eliminate architectural barriers and urban implementation plans).

If we want inclusive cities, we need to find adequate resources and effective operational tools to implement inclusive restyling in existing playgrounds and ensure the requirement of inclusion in all new play areas. In addition, stakeholder education should be promoted to strengthen the culture of inclusion in order to nurture new forms of creativity for playgrounds.

\section{References}

[1] Staccioli, G. 2017. "Il diritto al gioco di tutti i bambini: un percorso di lettura e filmografico." Rassegna bibliografica infanzia e adolescenza 4 (Sup.): 1-25.

[2] Autorità Garante per l'Infanzia e l'Adolescenza. 2018. Il diritto al gioco e allo sport dei bambini e dei ragazzi con disabilità. Documento di studio e di proposta. Report by 
the W.G. on Play and Sport for Children of the Italian Guarantor Authority for Children.

[3] Encarnação, P., Ray-Kaeser, S., and Bianquin, N., eds. 2018. Guidelines for Supporting Children with Disabilities' Play. Methodologies, Tools, and Contexts. Berlin: De Gruyter Poland Ltd.

[4] Suhonen, E., Takala, M., Alijoki, A., and Viljamaa, E. 2019. "7 Children with and without Disabilities in Finnish Early Childhood Education.” In Users' Needs Report on Play for Children with Disabilities. Parents' and Children's Views, 84-94.

[5] Lancerin, L., ed. 2003. Il verde è di tutti. Schede tecniche per la progettazione e la realizzazione di aree verdi accessibili e fruibili, editore. Padova: Regione Veneto.

[6] Ripat, J., and Becker, P. 2012. "Playground Usability: What Do Playground Users Say?" Occupational Therapy International 19: 144-53.

[7] Kim, Y.-G., Kim, H., and Maeng, S. 2018. "Characteristics of Inclusive Playground Guidelines." Journal of the Korean Institute of Landscape Architecture 46 (6): 75-84.

[8] Burke, J. 2013. "Just for the Fun of It: Making Playgrounds Accessible to All Children." World Leisure Journal 55 (1): 83-95.

[9] Stanton-Chapman, T. L., and Schmidt, E. L. 2017. "Creating an Inclusive Playground for Children of All Abilities: West Fork Playground in Cincinnati, Ohio." Children, Youth and Environments 27 (3): 124-37.

[10] Playright Children's Play Association. 2016. "Inclusive-Play-Space-Guide, Championing Better and More Inclusive Play Spaces in Hong Kong.” Accessed Oct. 31, 2020. https://playright.org.hk/wp-content/
uploads/2018/12/Playright-Inclusive-Play-Space-Guide.p df.

[11] Protti, C, and Bedetti, R. 2018. "Quanti sono i parchi gioco inclusivi in Italia?" Parchi per Tutti blog. Accessed Oct. 15, 2020. http://www.parchipertutti.com/parchiinclusivi-italia/.

[12] Laurìa, A., and Montalti, M. 2015. "Il Playground come laboratorio di creatività e di inclusione." Ri-Vista Research for Landscape Architecture 13 (1): 112-28.

[13] Siu, K. W. M., Wong, Y. L., and Lam, M. S. 2017. "Inclusive Play in Urban Cities: A Pilot Study of the Inclusive Playgrounds in Hong Kong." Procedia Engineering 198: 169-75.

[14] Riva, C. 2019. "Bambini con disabilità: non può bastare un'altalena in un parco." Accessed Oct. 31, 2020. https://www.superando.it/2019/05/30/non-puo-bastare-un altalena-in-un-parco/.

[15] Atmakur, S. 2013. "Focus: Playgrounds of Inclusion." Accessed Oct. 2020. https://www.unicef.org/sowc2013/focus_playgrounds_of _inclusion.html.

[16] Şensoy, G., and Inceoğlu, M. 2015. "Design Guide for Playgrounds." Journal of Civil Engineering and Architecture 9 (11): 1390-8.

[17] Buosi, F. 2015. “Creare l'inclusività. Il ruolo della Terapia Occupazionale nella progettazione dei parchi gioco per l'infanzia." Degree thesis in Occupational Therapy, University of Padova (Italy).

[18] Moore, A., and Lynch, H. 2015. "Accessibility and Usability of Playground Environments for Children under 12: A Scoping Review." Scandinavian Journal of Occupational Therapy 22 (5): 331-44. 